Physics

Physics Research Publications

\title{
Magnetic excitations of stripes near a quantum critical point
}

D. X. Yao

E. W. Carlson

D. K. Campbell 


\title{
Magnetic Excitations of Stripes near a Quantum Critical Point
}

\author{
D. X. Yao, ${ }^{1}$ E. W. Carlson, ${ }^{2}$ and D. K. Campbell ${ }^{1}$ \\ ${ }^{1}$ Department of Physics and Department of Electrical and Computer Engineering, Boston University, \\ Boston, Massachusetts 02215, USA \\ ${ }^{2}$ Department of Physics, Purdue University, West Lafayette, Indiana 47907, USA
}

(Received 6 January 2006; published 7 July 2006)

\begin{abstract}
We calculate the dynamical spin structure factor of spin waves for weakly coupled stripes. At low energy, the spin-wave cone intensity is strongly peaked on the inner branches. As energy is increased, there is a saddlepoint followed by a square-shaped continuum rotated $45^{\circ}$ from the low energy peaks. This is reminiscent of recent high energy neutron scattering data on the cuprates. The similarity at high energy between this semiclassical treatment and quantum fluctuations in spin ladders may be attributed to the proximity of a quantum critical point with a small critical exponent $\eta$.
\end{abstract}

DOI: 10.1103/PhysRevLett.97.017003

Strongly correlated electronic systems often exhibit some evidence of local electronic inhomogeneity, appearing in such diverse probes as neutron scattering, STM, $\mu \mathrm{sR}$, and NMR, among others [1-3]. In the high temperature superconductors, stripe structures are one possible microscopic realization of local electronic inhomogeneity. Stripes are electronic states which spontaneously break the rotational symmetry of the host crystal, the most ordered example being an interleaved, unidirectional modulation of both spin and charge density, as shown in Fig. 1. Recent neutron scattering experiments exploring the spin excitations in cuprates point to a universal high energy magnetic response in both yttrium barium copper oxide (YBCO) and (stripe-ordered) lanthanum barium copper oxide (LBCO) $[1,2,4]$, and it has been suggested that the universal high energy behavior may be connected to the superconductivity in these materials $[1,2]$. At low frequency, neutron scattering reveals four incommensurate spin peaks which disperse inward toward the $(\pi, \pi)$ point, converging at a resonance peak at intermediate energy. This is followed by a high energy square-shaped continuum in which the corners are rotated $45^{\circ}$ away from the direction of the low energy peaks $[1,2]$. The high energy response, in particular, has been attributed to the quantum excitations of spin ladders [1,5-7].

We find that the experimental results in LBCO at all energies are also consistent with semiclassical spin-wave excitations of weakly coupled stripes, i.e., for weak spin coupling across the charged domain walls in the spin pattern. We further suggest that the reason the quantum excitations of 2-leg ladders and the semiclassical spin waves studied here have such similar behavior at high energy is due to the proximity of a quantum critical point (QCP) with small critical exponent $\eta$. On both the ordered and disordered side of a QCP, above a certain characteristic frequency, the response is quantum critical, but for small $\eta$, the quantum critical behavior can look very much like the Goldstone behavior on the ordered side [8]. Calculations of quantum spin fluctuations due to high energy ladder behavior [5-7] are exploring this quantum
PACS numbers: 74.72.-h, 75.10.Jm, 75.30.Ds, 76.50.+g

critical regime at high energy, and can resemble much of the semiclassical behavior reported here.

In this Letter, we consider fully ordered static spin stripes, which are arrays of antiphase domain walls in an otherwise antiferromagnetic texture. We are interested solely in the response of the spin degrees of freedom and neglect the dynamics of the charge density which must peak on every domain wall. These charge degrees of freedom affect the spin degrees of freedom through a spatially modulated effective exchange integral. The undoped sys-

(a)

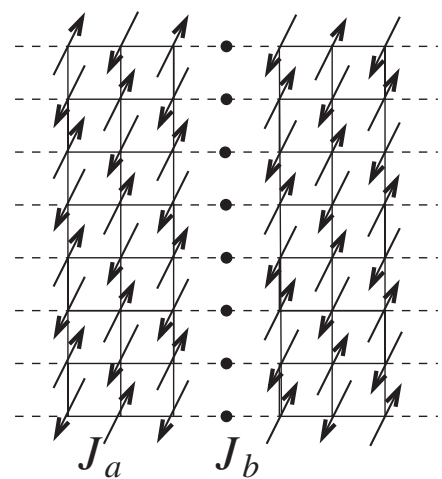

(b)

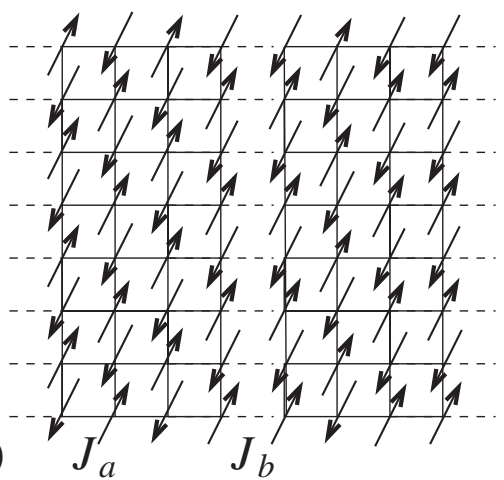

FIG. 1. (a) Site-centered stripes of spacing 4 viewed as weakly coupled 3-leg ladders. (b) Bond-centered stripes of spacing 4 viewed as weakly coupled 4-leg ladders. The coupling within each ladder is $J_{a}$, and the coupling between ladders is $J_{b}$. 
tem was shown to be quantitatively well described by the semiclassical spin waves of a 2D antiferromagnet [9]. When stripes form, the doping is topological, that is, doped holes create line dislocations in the antiferromagnetism, but in between the defects the antiferromagnet is more or less intact. Within this framework, it is reasonable to expect a semiclassical treatment to be applicable in a range of doping, as long as the ground state remains ordered. We take the coupling within an antiferromagnetic patch to be close to its full undoped value, and consider the effective spin coupling across a charged domain wall to be reduced, as illustrated by the dotted lines in Fig. 1. This corresponds to a Heisenberg model with modulated exchange integral on a square lattice, where each site represents a copper atom in the copper-oxygen plane:

$$
H=\frac{1}{2} \sum_{\left\langle\mathbf{r}, \mathbf{r}^{\prime}\right\rangle} J_{\mathbf{r}, \mathbf{r}^{\prime}}\left(\mathbf{S}_{\mathbf{r}} \cdot \mathbf{S}_{\mathbf{r}^{\prime}}\right),
$$

where $J_{\mathbf{r}, \mathbf{r}^{\prime}}$ is the exchange coupling. Nearest neighbor couplings are positive $J_{\mathbf{r}, \mathbf{r}^{\prime}}=J_{a}>0$ within each antiferromagnetic patch. Couplings across domain walls depend on whether stripes are site- or bond-centered, as explained below. We work in units where $\hbar=1$.

"Site-centered" stripes have domain walls which are centered on the sites of the square lattice (i.e. on the copper sites), leading to an antiferromagnetic effective coupling $J_{\mathbf{r}, \mathbf{r}^{\prime}}=J_{b}>0$ across the domain walls. For weak coupling across the domain walls $J_{b} \ll J_{a}$, the system is close to the regime of coupled 3-leg ladders, as shown in Fig. 1(a). "Bond-centered" stripes have domain walls which are centered between the sites, leading to a ferromagnetic effective coupling $J_{\mathbf{r}, \mathbf{r}^{\prime}}=J_{b}<0$ across the domain walls [10]. For weak coupling across the domain walls $\left|J_{b}\right| \ll J_{a}$, the system is close to coupled 4-leg ladders [11], as shown in Fig. 1(b). When $J_{b}$ is sufficiently large, both cases presumably have a long-range ordered ground state [12] with low energy Goldstone behavior, but there is a quantum critical point at small $J_{b}$ beyond which the physics scales to that of decoupled ladders. While the ground state of coupled oddleg $s=1 / 2$ ladders is ordered with any finite coupling and therefore $J_{b}^{\text {crit }}=0$, coupled even-leg ladders have a finite value for the quantum critical point, $\left|J_{b}^{\text {crit }}\right|>0$.

We use semiclassical linearized spin-wave theory and Holstein-Primakoff bosons, a standard procedure described elsewhere [13-15], in order to calculate the spinwave excitation spectrum and the zero-temperature dynamical structure factor,

$$
S(\mathbf{k}, \omega)=\sum_{f} \sum_{i=x, y, z}\left|\left\langle f\left|S^{i}(\mathbf{k})\right| 0\right\rangle\right|^{2} \delta\left(\omega-\omega_{f}\right),
$$

which is related to the expected neutron scattering intensity.

Figure 2 shows the intensity of the dynamical structure factor for the site-centered stripes of Fig. 1(a) as a function of frequency for weak coupling strength $J_{b}=0.05 J_{a}$. Figure 3 shows the results for the bond-centered stripes
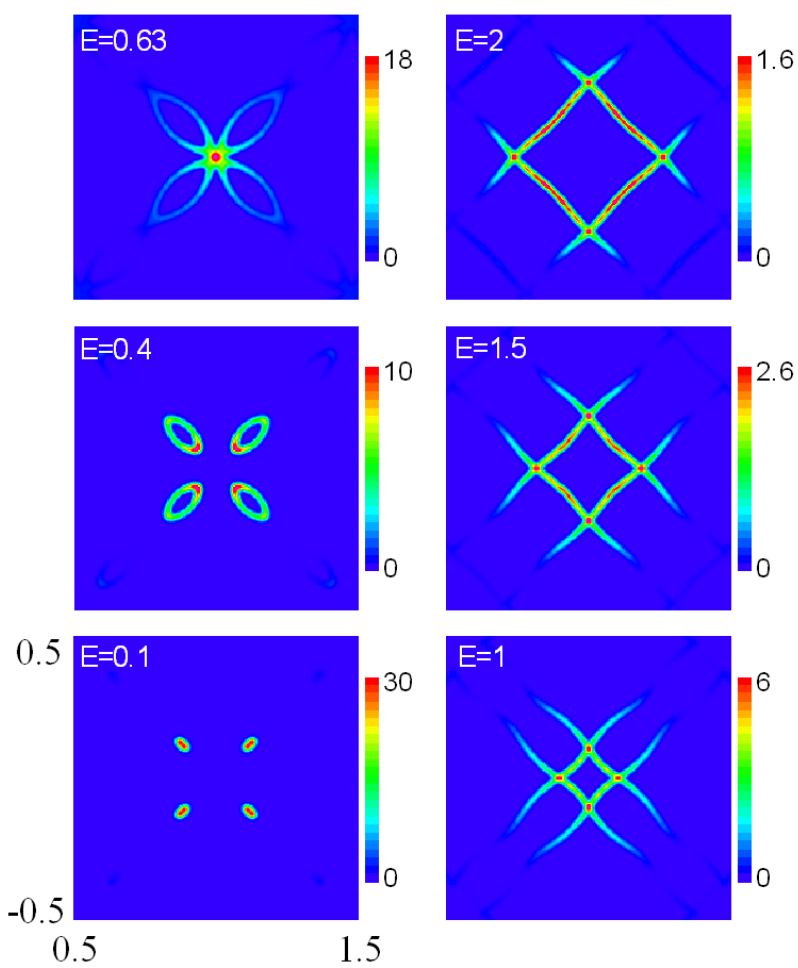

FIG. 2 (color online). Constant energy cuts, integrated within an energy window of $\pm 0.05 J_{a} S$ for vertical, site-centered stripes of spacing 4 at $J_{b}=0.05 J_{a}$ in the magnetic Brillouin zone. The energy $E$ is in units of $J_{a} S$. Results are shown for twinned stripes.

of Fig. 1(b) for weak coupling strength $J_{b}=-0.09 J_{a}$. Both coupling strengths have ordered ground states, as discussed below. The results are rather similar for siteand bond-centered stripes [5], with the main difference being that while the satellite peaks are too weak to be visible at low energy in the site-centered case, they are visible (although faint) at low energy in the bond-centered case. At low energies, four strong incommensurate peaks are visible, which disperse inward toward $(\pi, \pi)$ as energy is increased. Although a spin-wave cone must emanate from each magnetic reciprocal lattice vector due to Goldstone's theorem, the intensity is not necessarily uniform. For weak spin coupling across the charged domain wall, $\left|J_{b}\right| \ll\left|J_{a}\right|$, we find that the intensity is strongly peaked on the inner branches emanating from $(\pi, \pi \pm$ $\pi / 4)$, and the twinned IC peaks at $(\pi \pm \pi / 4, \pi)$, that is, the side of the cones that is nearest $(\pi, \pi)$ [16]. This situation is reversed for strong $\left|J_{b}\right| \gg\left|J_{a}\right|$, where the intensity in the spin-wave cones is strongest on the outer branches emanating from the same points. One mystery about the low energy neutron scattering results in cuprates has been that spin-wave cones are not observed, but rather, the intensity disperses toward $(\pi, \pi)$. We have shown here that this is consistent with semiclassical spin waves of weakly coupled stripes.

Upon increasing the energy, there is a resonance peak apparent at $E_{\text {res }}=0.63 J_{a} S$ and $(\pi, \pi)$ in Fig. 2, which is a 

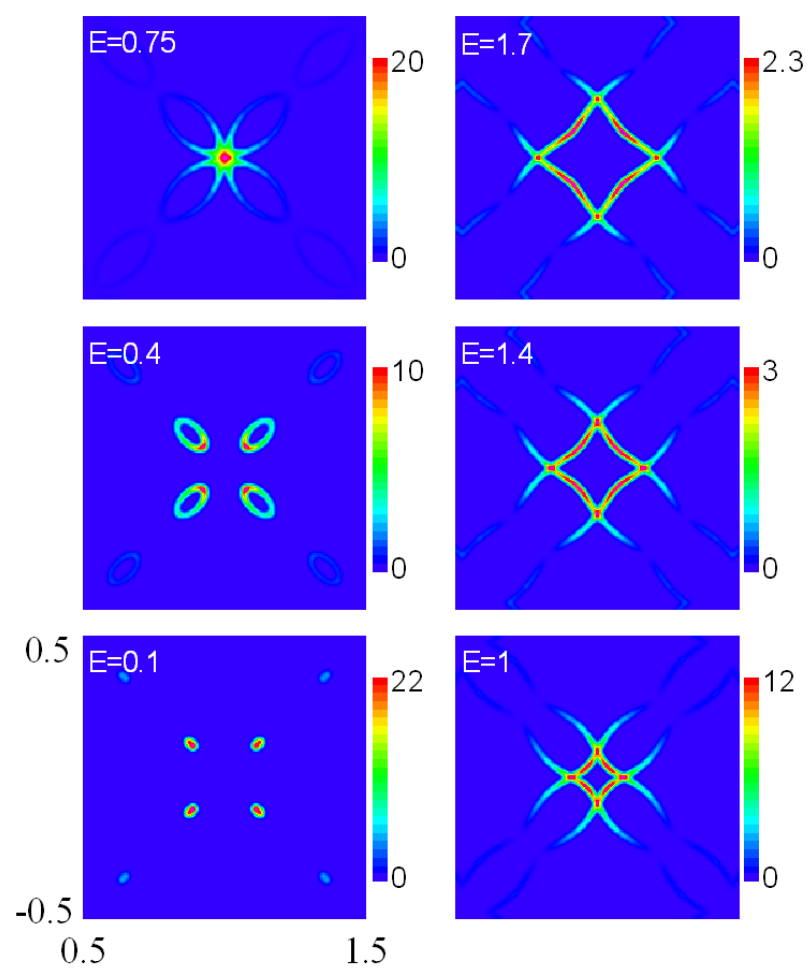

FIG. 3 (color online). (Color online) Constant energy cuts, integrated within an energy window of $\pm 0.05 J_{a} S$ for vertical, bond-centered stripes of spacing 4 at $J_{b}=-0.09 J_{a}$ in the magnetic Brillouin zone. The energy $E$ is in units of $J_{a} S$. Results are shown for twinned stripes.

saddle point at which the integrated intensity $S(\omega)$ has a maximum (see Fig. 4). In Fig. 3, the resonance is at $E_{\text {res }}=$ $0.75 J_{a} S$. The energy scale of this resonance increases with increasing $\left|J_{b} / J_{a}\right|$. Using $E_{\text {res }}=50-60 \mathrm{meV}$ from Ref. [1], this yields $J_{a}=160-190 \mathrm{meV}$ in Fig. 2 and $J_{a}=$ $130-160 \mathrm{meV}$ in Fig. 3. This is reasonable given a value of $J_{a}=140 \mathrm{meV}$ in undoped $\mathrm{La}_{2} \mathrm{CuO}_{4}[1]$, and it is consistent with other theoretical estimates on LBCO $[5,6]$. For weak enough coupling $J_{b}$, the dispersion above the saddle point is highly anisotropic, giving rise to a rotated squareshaped continuum above the resonance peak, much like what is seen in LBCO [1].

Figure 4 shows the single magnon contribution to the momentum-integrated structure factor $S(\omega)$ for sitecentered stripes of Fig. 2, and reveals the effect of the saddle point in the acoustic band. The broad peak in $S(\omega)$ at $E_{\text {res }}=0.63 J_{a} S$ is due to the saddle point. Qualitatively, the results at high energy are similar to the one-triplon calculations based on coupled 2-leg ladders $[5,6]$. Contributions from the first optical band can be seen above $E=2.2 J_{a} S$.

We find that we can describe both the low energy and the high energy neutron scattering data on LBCO [1] within the semiclassical framework of spin waves, for weak spin coupling across the charge domain walls. This brings up two questions: (1) Why are the semiclassical results so similar to the quantum critical behavior in other models of

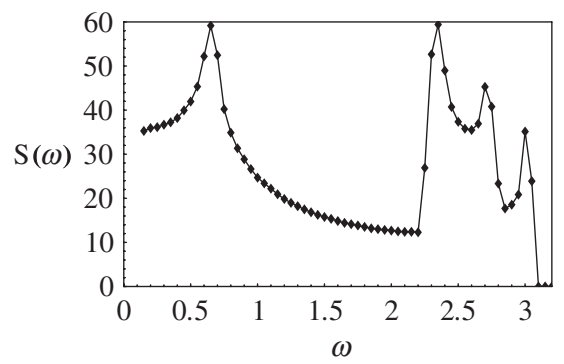

FIG. 4. $S(\omega)$ for site-centered stripes of spacing 4 at $J_{b}=$ $0.05 J_{a}$ over the magnetic Brillouin zone. The frequency $\omega$ is in units of $J_{a} S$. The broadening $\Delta \omega$ is of $\pm 0.05 J_{a} S$.

stripes [17], and (2) Why is the high energy response of YBCO so similar to that of stripe-ordered LBCO, when YBCO does not show evidence of long-range magnetic order? Concerning the first question, the similarity may be due to the proximity to a quantum critical point with small critical exponent $\eta$ [8]. The elementary excitations of the system fundamentally change in character across the quantum critical point, and for $\eta>0$ the structure factors in the ordered and quantum critical regimes have analytically distinct forms. However, the spectrum is distributed in a similar way for $\eta \geqslant 0$. In fact, as $\eta \rightarrow 0$, the two cases become indistinguishable. We are considering a $2 \mathrm{D}$ quantum transition to distinct 3-leg or 4-leg ladders, and the universality class is the classical $(2+1)$-dimensional Heisenberg model. Since then the critical exponent $\eta=$ 0.037 is small [19], for all practical purposes there may be little distinction between the classical and quantum critical cases. However, sufficient resolution in line shapes can in principle distinguish: whereas weakly interacting spin waves produce a Lorentzian line shape, quantum criticality produces a power law cusp $[8,10,20]$. Concerning the second question, the gap in YBCO indicates that it must be on the disordered side of the QCP. However, the fact that YBCO's high energy response is so similar to the Goldstone modes calculated here is likely an indication that YBCO is close to the QCP.

Figure 5(a) shows the crossover energy scales for coupled odd-leg ladders as a function of interladder coupling $J_{b}$. In this case, the quantum critical point is at $J_{b}^{\text {crit }}=$ 0 , where the system breaks up into independent odd-leg ladders for $S=1 / 2$. At $J_{b}=0$, the low frequency behavior is controlled by the $c=1$ Wess-Zumino-Witten (WZW)

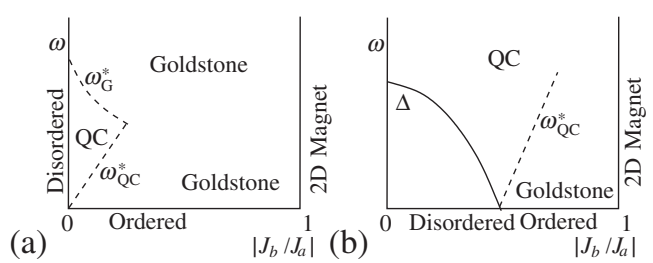

FIG. 5. Crossovers for coupled ladders as a function of interladder coupling $J_{b}$ for both odd-leg ladders (a) and even-leg ladders (b). 
model, and the high frequency behavior scales to that of weakly interacting Goldstone modes [12]. Away from the QCP, the ground state is ordered and the lowest energy response is due to Goldstone modes. Quantum critical behavior obtains above a lower crossover $\omega_{\mathrm{QC}}^{*} \propto\left(J_{b}\right)^{\nu z}$ [20], and the high energy Goldstone behavior of decoupled odd-leg ladders is recovered above a higher crossover energy scale $\omega_{\mathrm{G}}^{*} \propto \frac{v}{\xi} \propto v e^{-W_{\text {eff }}}$ [12], where the effective width of ladders $W_{\text {eff }}$ increases as $J_{b}$ increases. The two energy scales $\omega_{\mathrm{QC}}^{*}$ and $\omega_{\mathrm{G}}^{*}$ approach each other with increasing $J_{b} / J_{a}$, so that beyond a certain point the WZW behavior is squeezed out entirely [12], and in this region our approach is well justified. Moreover, because the critical exponent $\eta=0.037$ is small, even the quantum critical WZW region can resemble the Goldstone behavior [8].

Figure 5(a) shows the gap $\Delta$ and the quantum critical energy scale $\omega_{\mathrm{QC}}^{*}$ for coupled even-leg ladders as a function of $J_{b}$ [21]. In this case, the quantum phase transition is at finite coupling $J_{b}^{\text {crit }}>0$. To find the quantum critical point for coupled 4-leg ladders, we use the stochastic series expansion quantum Monte Carlo (QMC) method [22]. Using finite size scaling on the spin stiffness and the Binder ratio, we find that $J_{b}^{\text {crit }}=0.076(3) J_{a}$ for weakly coupled 4-leg spin-1/2 ladders, consistent with Ref. [23]. (For weakly coupled 2-leg ladders, $J_{b}^{\text {crit }} \approx 0.25 J_{a}-0.3 J_{a}$ $[21,24]$.) Our spin-wave calculations presented in Fig. 3 correspond to weakly coupled 4-leg ladders, close to but on the ordered side of the QCP. Using QMC on this configuration for systems up to size $64 \times 32$, we find that the sublattice magnetization is $m_{z}=0.143$ ( $47 \%$ of 0.307 for the 2D antiferromagnet), confirming that the system is ordered at this coupling.

Quenched disorder in the form of dopant atoms introduces disorder in the ladder widths, positions, and couplings. Weak disorder is irrelevant at the critical point of a $(2+1)$-dimensional Heisenberg model, and although disorder can lower the transition temperature (a finite ordering temperature is possible with any weak $c$-axis coupling [9]), the critical exponent $\eta$ is unchanged. Doping may even induce a QCP through the introduction of disorder, doping the ladders, or by inducing dynamical stripes with fluctuating charge.

In conclusion, we have studied the magnetic excitations of ordered stripes in the proximity of a quantum critical point. For weak spin coupling across the charged domain walls, the low energy spin-wave cones have weight which disperses inward toward $(\pi, \pi)$, in agreement with experiment. At higher energies, the semiclassical excitations develop saddle points with steep dispersions. The saddle point acts like a "resonance peak" in that there is extra intensity due to the saddle point, and also due to stripe twinning. (However, our model does not address the observed increase in intensity of the resonance peak as superconductivity onsets.) At energies above the saddle point, there is a slowly dispersing square-shaped continuum. The proximity to a quantum critical point (either to decoupled 3-leg ladders or to weakly coupled 4-leg ladders) with small critical exponent $\eta$ implies that quantum critical behavior (and therefore quantum ladder calculations at high energy) can strongly resemble the semiclassical calculations presented here.

It is a pleasure to thank A. Sandvik, S. Kivelson, and J. Tranquada for helpful discussions. This work was supported by Boston University (D.X. Y. and D. K. C.), and by the Purdue Research Foundation (E. W. C.).

[1] J. M. Tranquada et al., Nature (London) 429, 534 (2004).

[2] S. M. Hayden et al., Nature (London) 429, 531 (2004).

[3] For a review, see, e.g., E. W. Carlson et al., in The Physics of Superconductors, edited by J. Ketterson and K. Benneman (Springer-Verlag, Berlin, 2004), Vol. II, and references therein.

[4] C. Stock et al., Phys. Rev. B 71, 024522 (2005).

[5] M. Vojta and T. Ulbricht, Phys. Rev. Lett. 93, 127002 (2004).

[6] G. S. Uhrig, K. P. Schmidt, and M. Grüninger, Phys. Rev. Lett. 93, 267003 (2004).

[7] G. Seibold and J. Lorenzana, Phys. Rev. Lett. 94, 107006 (2005).

[8] S. A. Kivelson et al., Rev. Mod. Phys. 75, 1201 (2003).

[9] S. Chakravarty, B. I. Halperin, and D. R. Nelson, Phys. Rev. Lett. 60, 1057 (1988).

[10] J. M. Tranquada et al., Phys. Rev. B 55, R6113 (1997).

[11] Coupled 2-leg ladders have been considered in Refs. [5,6]. We are considering here an effective theory of the behavior of the net moment on each site, with some smooth envelope for the moments which must have a node at each domain wall. This produces a small but finite moment even on sites right next to the domain walls, and so we model bond-centered stripes as weakly coupled 4-leg ladders.

[12] S. Chakravarty, Phys. Rev. Lett. 77, 4446 (1996).

[13] E. W. Carlson, D. X. Yao, and D. K. Campbell, Phys. Rev. B 70, 064505 (2004).

[14] A. Auerbach, Phys. Rev. Lett. 72, 2931 (1994).

[15] F. Krüger and S. Scheidl, Phys. Rev. B 67, 134512 (2003).

[16] D. X. Yao, E. W. Carlson, and D. K. Campbell, cond-mat/ 0603829 [Phys. Rev. B (to be published)].

[17] Models pointing to quantum behavior at high energy include weakly coupled 2-leg ladders $[1,5,6]$, a phenomenological quantum lattice model [18], and Gutzwiller projections of the Hubbard model [7].

[18] M. Vojta and S. Sachdev, J. Phys. Chem. Solids 67, 11 (2006).

[19] P. Sengupta, A. W. Sandvik, and R. R. P. Singh, Phys. Rev. B 68, 094423 (2003).

[20] S. Sachdev, Science 288, 475 (2000).

[21] S. Gopalan, T. M. Rice, and M. Sigrist, Phys. Rev. B 49, 8901 (1994).

[22] A. W. Sandvik and J. Kurkijärvi, Phys. Rev. B 43, 5950 (1991).

[23] Y. J. Kim et al., Phys. Rev. B 60, 3294 (1999).

[24] J. Tworzydlo et al., Phys. Rev. B 59, 115 (1999). 